The following paper posted here is not the official IEEE published version. The final published version of this paper can be found in the Proceedings of the IEEE International Conference on Geoscience and Remote Sensing Symposium (2006 : Denver, Colorado):pp. 71-74

Copyright @ 2006 IEEE.

Personal use of this material is permitted. However, permission to reprint/republish this material for advertising or promotional purposes or for creating new collective works for resale or redistribution to servers or lists, or to reuse any copyrighted component of this work in other works must be obtained from the IEEE. 


\section{Structural Parameter Estimation of Australian Flora with a Ground-based Polarimetric Radar Interferometer}

\author{
Zheng-Shu Zhou \\ School of Electrical \& Electronic Engineering \\ The University of Adelaide \\ Adelaide, Australia \\ zszhou@eleceng.adelaide.edu.au
}

\author{
Shane R. Cloude \\ AEL Consultants \\ Cupar, Scotland, UK \\ scloude@ieee.org
}

\begin{abstract}
The application of polarimetric SAR interferometry technology is extended to ground based radar imaging (GBPOLInSAR), which has a limited field of view but provides wide band coverage. It also provides for easier deployment of broadband and multi-baseline techniques, from which we can estimate vegetation structure and extinction propagation using model based techniques. This micro-scale multi-parameter combination with good temporal resolution is a unique feature of groundbased sensors.

In this paper we present both polarimetric and interferometric coherence calibration results for such a GB-POLInSAR system we have developed at the University of Adelaide. We then show an initial study of height estimation of Australian native plants based on the coherence parameter retrieval models of the polarimetric SAR interferometry technique using the broadband GB-POLInSAR system.
\end{abstract}

Keywords-POLInSAR; ground-based SAR; oriented-volumeover-ground model (OVOG); vegetation parameter retrieval

\section{INTRODUCTION}

The combination of imaging radar polarimetry and interferometry (called POLInSAR) [1] is a promising new technology for the quantitative remote sensing of vegetated land surfaces with important applications in the remote measurement of vegetation properties such as forest height and biomass as well as emerging applications in agriculture, snow/ice thickness monitoring and urban height and structure applications [2]. It has been developed mainly for applications in space and airborne SAR systems and little attention has been paid to its potential role in ground-based observations. We consider application of this technology to ground based radar imaging (GB-POLInSAR) [3]. Such a facility has a limited field of view but provides wide band coverage with none of the problems of poor temporal sampling associated with airborne and space-borne sensors. Moreover, GB-POLInSAR has several potential advantages over existing ground based sensing methods $[4,5]$. As an instrument for robust quantitative studies of vegetation parameters, it can potentially be used to monitor vegetation dynamics and extract parameters such as growth rates, plant water content and surface moisture with high spatial and temporal resolution [3]. There is also the potential to study sub-canopy surface parameters such as soil moisture. This micro-scale multi-parameter combination with good temporal resolution is a unique feature of ground-based sensors. GB-POLInSAR systems also provide the potential for extracting 3-D structural information much faster than full 3-D scans, which is important for vegetation applications in the field, where temporal motion effects can defocus 3-D images. At the same time, it is important for developing new algorithms and as a validation tool for POLInSAR applications to space borne and airborne SAR sensors.

In this paper we show the system performance validation on both polarimetric and interferometric coherence calibration for such a GB-POLInSAR system. We then review the model based vegetation biophysical parameter retrieval algorithm. In particular we show an analysis of the height estimation for the Australian native plant Syzygium australe using our broadband GB-POLInSAR system.

\section{SyStem VALIDATION}

\section{A. Polarimetric Calibration}

Making use of our broadband GB-POLInSAR system [3], we carried out some experiments with a few of the standard reflectors for radar polarimetry and interferometry to demonstrate the polarimetric performance and interferometric coherence of this system.

A modified polarimetric radar cross section (RCS) calibration technique using two orientations of the dihedral corner reflectors as calibration targets was introduced [6]. This method is valid for any monostatic or quasi-monostatic radar system. We have carried out some calibration tests in the anechoic chamber. A dihedral corner reflector in multiple orientations was employed as a polarimetric calibrator for the system. From the theoretical value of two calibrators and measured data, we recovered the calibration coefficients. Using these calibration coefficients, the calibrated RCSs for typical orientated dihedrals can be calculated, which are shown in Fig. 1. From the figure, we find the improvements after calibration to be rather considerable and satisfactory for the intended

This work was partially supported by the Defence Science and Technology Organisation (DSTO), Australia. 
application, especially in the phase term. The system demonstrates good stability and repeatability with cross-pol isolation better than $-25 \mathrm{~dB}$ and phase and amplitude imbalances less than 5 degree and $0.5 \mathrm{~dB}$ across the band, respectively.
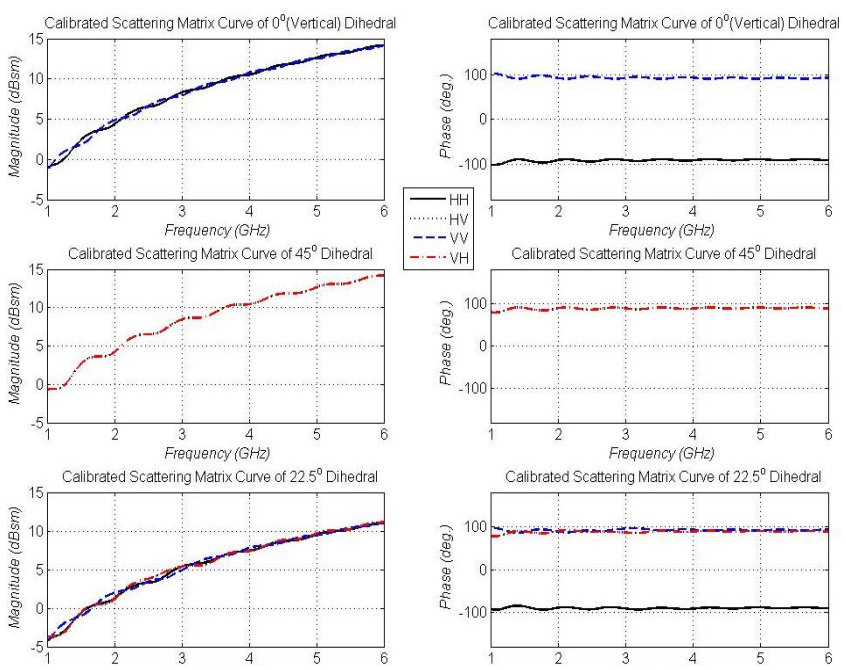

Figure 1. Calibration results for dihedral at three orientation angles.

\section{B. Interferometric Coherence Calibration}

Furthermore, we performed measurements for system interferometric coherence calibration. A dihedral corner reflector as a calibrator was located $4.7 \mathrm{~m}$ down range from the antenna and $0.38 \mathrm{~m}$ above floor level. The antenna was placed on a vertical mast and looked down at the target with an incidence angle of 70.8 degrees. Interferometric measurements with full polarization components were carried out with baseline $10 \mathrm{~cm}$ from height $2.05 \mathrm{~m}$ to $2.15 \mathrm{~m}$. Coherence is then obtained by frequency averaging. For calibrating interferometric phase, we compensate the phase of slave signal in order to match the master signal due to the displacement of vertical baseline. Therefore, interferometric phase zero is obtained as the reference level for height estimation of vegetation. Fig. 2 shows interferometric coherences and phases of co-polarization components for the dihedral. Amplitude of coherence is better than 0.995 and accuracy of interferometric phase is better than 15 degrees across $3 \mathrm{GHz}$ to $6 \mathrm{GHz}$.
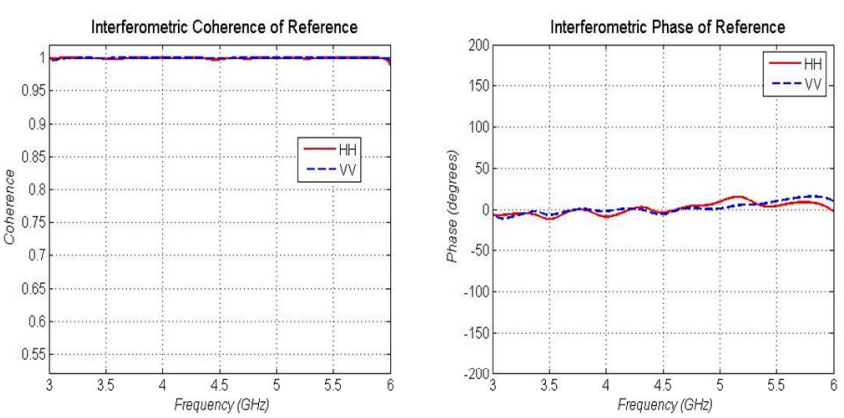

Figure 2. Interferometric coherence calibration.

\section{Vegetation Retrieval Models For GB-POLInSAR}

The penetration of microwaves into and through vegetation at longer wavelengths makes volume decorrelation an important issue for interferometry studies. As a source of phase variance it contains physical information about the vertical structure of the volume scatterers. In general, interferometric coherence can be decomposed into various contributions as shown in (1) $[2,7]$

$$
\tilde{\gamma}=e^{i \phi\left(z_{o}\right)} \gamma_{\text {snr }} \gamma_{t} \tilde{\gamma}_{v}
$$

where $\gamma_{s n r}$-noise coherence, $\gamma_{t}$-temporal coherence, $\gamma_{v}$ volume coherence. Considering extinction inside the uniform vegetation layer, volume coherence can be described as [2]

$$
\widetilde{\gamma}_{v}=\gamma e^{i \varphi}=\frac{2 \sigma e^{i \phi\left(z_{o}\right)}}{\cos \theta_{o}\left(e^{2 \sigma h_{v} / \cos \theta_{o}}-1\right)} \int_{0}^{h_{v}} e^{i k_{z} z^{\prime}} e^{\frac{2 \sigma z^{\prime}}{\cos \theta_{o}}} d z^{\prime}
$$

Here there are two observations and three unknowns (mean extinction, vegetation height, and ground topography). Therefore a single channel interferometer is underdetermined for parameter estimation. For short vegetation such as crops or plants we have differential extinction to consider. We then get a measurable difference between the coherences in different polarisations which in turn can be related to physical parameters as [8]:

$$
\begin{aligned}
& \tilde{\gamma}_{1}=\frac{2 \sigma_{h} e^{i \phi\left(z_{o}\right)}}{\cos \theta_{o}\left(e^{2 \sigma_{h} h_{v} / \cos \theta_{o}}-1\right)} \int_{0}^{h_{v}} e^{i k_{z^{\prime}} z^{\prime}} e^{\frac{2 \sigma_{h^{\prime} z^{\prime}}}{\cos \theta_{o}}} d z^{\prime} \\
& \widetilde{\gamma}_{2}=\frac{\left(\sigma_{h}+\sigma_{v}\right) e^{i \phi\left(z_{o}\right)}}{\cos \theta_{o}\left(e^{\left(\sigma_{h}+\sigma_{v}\right) h_{v} / \cos \theta_{o}}-1\right)} \int_{0}^{h_{v}} e^{i k_{z^{\prime}} z^{\prime}} e^{\frac{\left(\sigma_{h}+\sigma_{v}\right) z^{\prime}}{\cos \theta_{o}}} d z^{\prime} \\
& \widetilde{\gamma}_{3}=\frac{2 \sigma_{v} e^{i \phi\left(z_{o}\right)}}{\cos \theta_{o}\left(e^{2 \sigma_{v} h_{v} / \cos \theta_{o}}-1\right)} \int_{0}^{h_{v}} e^{i k_{z^{\prime}} z^{\prime}} e^{\frac{2 \sigma_{v} z^{\prime}}{\cos \theta_{o}}} d z^{\prime}
\end{aligned}
$$

where $\sigma_{v} \gg \sigma_{h}$ (strong uniaxial component) for an oriented volume model [8]. Now there are six observations with four unknowns $\left(\sigma_{h}, \sigma_{v}, h_{v}, \phi\right)$, which can be inverted. But for short vegetation we need to consider the influence of surface scattering [2]. To motivate the development of such a system for bio-/geo-physical parameter estimation of short Australian native plants, we now turn to consider the robustness of coherence based retrieval models for the wide band GBPOLInSAR.

The data set selected for oriented-volume-over-ground (OVOG) model investigation $[2,8]$ is an indoor measurement (hence free of temporal decorrelation) of an Australian native plant - the Lilly Pilly (scientific name: Syzygium australe). Fig. 3 shows an image of the test sample and its setup in the anechoic chamber. The sample consists of a group of Lilly Pillies with a maximum height of $0.45 \mathrm{~m}$ planted in a round container with diameter $0.5 \mathrm{~m}$. They are evergreen plants with dense glossy green leaves. 

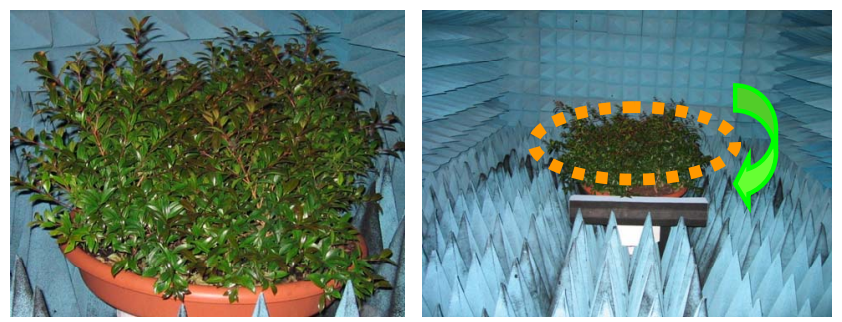

Figure 3. Test vegetation sample and its setup in the anechoic chamber.

The vegetation sample is placed on a rotating turntable so that measurements can be made over 360 degrees of azimuth for a given angle of incidence. The antenna beamwidth is such that the sample is uniformly illuminated by the transmitter. In this experiment there were 121 azimuth steps of 3 degrees. At each position the frequency is stepped across the frequency range 3 to $6 \mathrm{GHz}$ (in $6.25 \mathrm{MHz}$ steps) and the elevation baseline is $10 \mathrm{~cm}$ from $2.05 \mathrm{~m}$ to $2.15 \mathrm{~m}$. In this way interferometric analysis can be performed with a minimum baseline of $5 \mathrm{~cm}$ as well. Note finally that the focus for the sensor (zero phase position for interferometry) is located around $2 \mathrm{~cm}$ lower than the ground surface of the sample which is $40 \mathrm{~cm}$ above the floor level of the chamber. Hence the ground phase parameter $\phi$ in (1) will not be zero and will change with frequency and baseline. In this particular case, the ground distance from antenna to the target centre is $4.7 \mathrm{~m}$. Therefore, the incident angle is 70.8 degrees and the corresponding angle increment is 1.08 degrees.

The processing of the wide band signals starts with the two calibrated complex signals s1, s2 from position $\theta$ and $\theta+\Delta \theta$ as given by

$$
s_{1}=E_{\theta}^{p q}(f) \quad s_{2}=E_{\theta+\Delta \theta}^{p q}(f) .
$$

The wide band interferogram is then formed from the product of common spectral band filtered and phase offset signals as

$$
S_{1} \cdot s_{2}^{*}=E_{\theta}^{p q}(f) \cdot \operatorname{conj}\left(E_{\theta+\Delta \theta}^{p q}\left(f-\frac{f \Delta \theta}{\tan \theta}\right)\right) e^{-i \frac{4 \pi f \Delta \theta}{c \sin \theta} z_{o}} .
$$

Finally the complex coherence for polarization combination $p q$ and frequency $f$ is calculated as

$$
\tilde{\gamma}_{p q}(f)=\frac{\left\langle s_{1} s_{2}^{*}\right\rangle}{\sqrt{\left\langle s_{1} s_{1}^{*}\right\rangle\left\langle s_{2} s_{2}^{*}\right\rangle}}
$$

where $\langle\cdots\rangle$ denotes averaging which is made over 360 degrees of azimuth coverage and bandwidth smoothing [3,9]. Fig. 4 shows the resulting variation of interferometric coherence from (8) vs frequency. The ground phase has been normalized to zero for all frequencies. The vegetation bias increases with frequency at the same time as the coherence magnitude reduces. This is characteristic of the presence of volume decorrelation, the physical mechanism supporting the OVOG model. The coherence of vegetated land surfaces depends on several import vegetation biophysical and surface parameters.
The most important of these are the mean vegetation height, mean extinction coefficients and the true ground topographic phase. Here we concentrate on validation of height estimation from coherence.

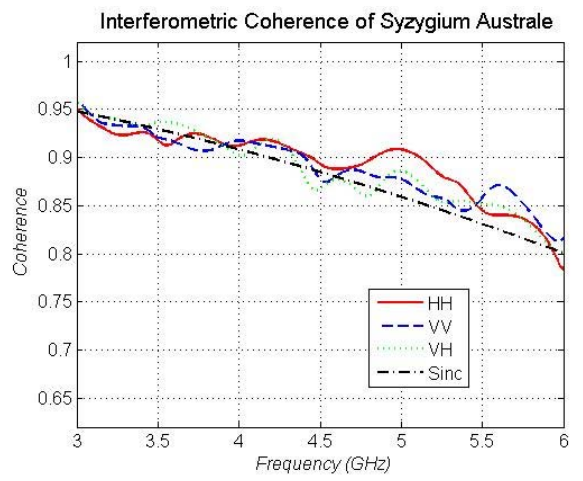

Figure 4. Variation of interferometric coherence vs frequency.

\section{Flora Height Estimation}

Vegetation height can be estimated by using a polarization channel that scatters from the top of the canopy [7, 10]. Usually the $\mathrm{VH}$ channel is used due to its domination by volume scattering. Note however that the $\mathrm{VH}$ phase centre can lie anywhere between half the height and the top of the canopy itself. When the vegetation canopy occurs over the full height, the phase centre can be at half height of vegetation for low density due to small extinction and then moves up to the top of the canopy for dense vegetation with high extinction. For trees with a high and thin canopy, extinction is small and the phase centre is high. This ambiguity is inherent to volume scattering and so we need to employ surface-volume model based correction methods as shown above. We plot the complex coherence ambiguity as coherence region boundary for Syzygium australe against frequency in Fig. 5. With increasing frequency, the phase and phase variation go up gradually and interferometric coherence decreases. Figure 6 shows the height estimated from interferometric phase only.

From the estimated vegetation height in Fig. 6, we find that the height of plants is underestimated. To improve the estimate we employ a mixed coherence/phase algorithm as described in [11]. The height of plant is then defined as:

$$
\hat{h}=\hat{h}_{1}+\varepsilon \hat{h}_{2} \approx h_{\text {true }}
$$

where $h_{l}$ is the phase based height estimate defined as

$\hat{h}_{1}=\left(\phi_{h_{v}}-\phi_{g}\right) / k_{z}<h_{\text {true }}, \hat{\phi}_{h_{v}}$-coherence phase, $\phi_{g}$-ground level and $h_{2}$ is a coherence correction defined as

$$
\left|\gamma_{h_{v}}\right| \Rightarrow \hat{h}_{2} \text { by }\left|\gamma_{h_{v}}\right|=\sin c \frac{k_{z} h_{2}}{2}
$$

When $\varepsilon=0.4$ is used in (9), as suggested in [11], the compensated height of Syzygium australe is shown in Fig. 7. An improvement in estimated height is observed but still slightly underestimated at lower frequencies and overestimated at higher frequencies. 

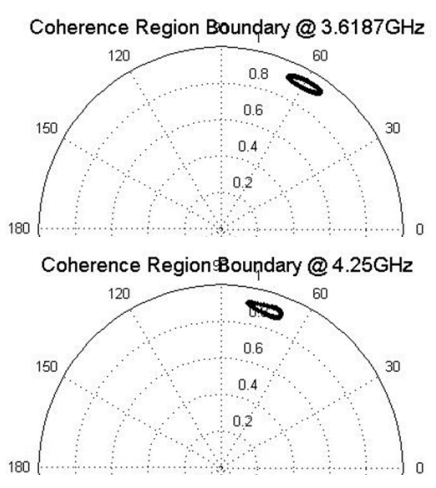

Coherence Region Boundary @ 4.8688GHz

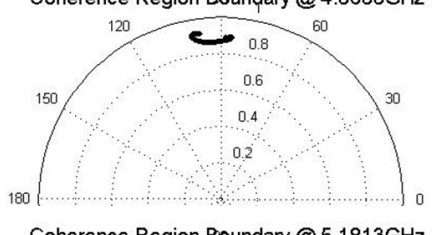

Coherence Region Boundary @ 5.1813GHz

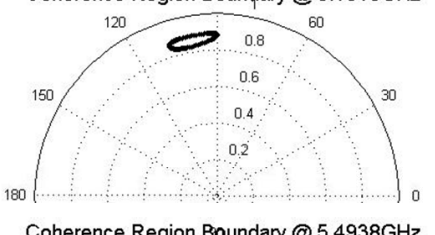

Coherence Region Boundary @ 5.4938GHz

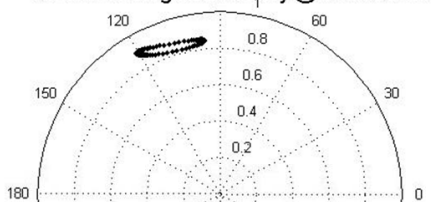

Figure 5. Interferometric cohrence region boundary against frequency.

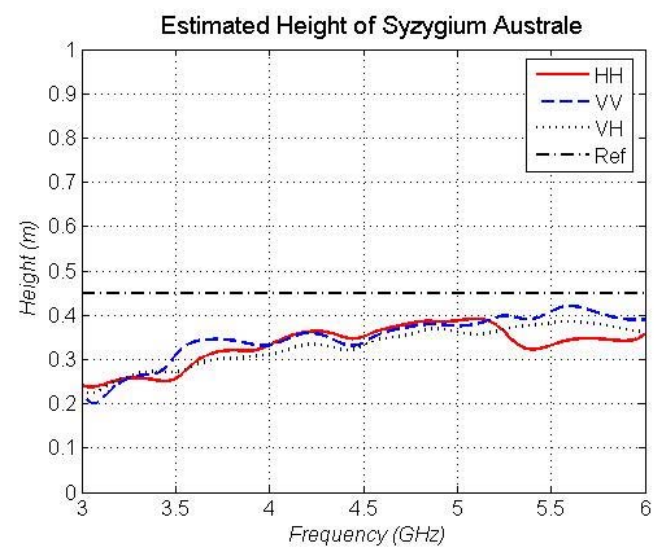

Figure 6. Phase based height estimate of Syzygium Australe.

\section{CONCLUSIONS}

In this paper a new GB-POLInSAR system is introduced and a study made to demonstrate the robustness of coherence based parameter retrieval models for application in a GB environment. Network-Analyzer-based GB-POLInSAR systems provide for easier deployment of broad-band and multi-baseline techniques, from which we proposed to estimate vegetation structure using model based techniques for an
Australian native plant - Syzygium australe. With coherence height compensation, the estimation accuracy for vegetation height is better than $10 \mathrm{~cm}$.

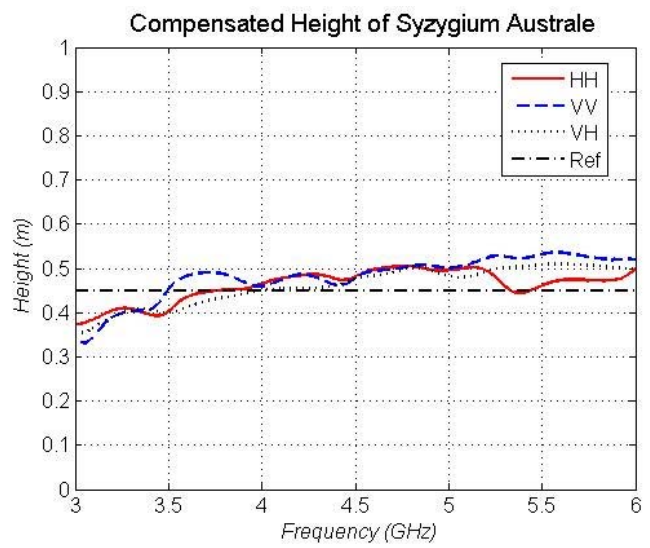

Figure 7. Compensated height of Syzygium Australe (equation 9).

\section{ACKNOWLEDGMENT}

We would like to thank Professor B. D. Bates of the University of Adelaide for his support and the constructive comments on this paper.

\section{REFERENCES}

[1] S. R. Cloude, K. P. Papathanassiou, "Polarimetric SAR interferometry," IEEE Trans. Geosci. Remote Sensing, vol. 36, pp. 1551-1565, September 1998.

[2] S. R. Cloude, K. P. Papathanassiou, "A 3-stage inversion process for polarimetric SAR interferometry," IEE Proc. Radar Sonar Navigation, vol. 150, pp. 125-134, June 2003.

[3] Z.-S. Zhou, S. R. Cloude, "The development of a ground based polarimetric SAR interferometer (GB-POLInSAR)," in Proc. of IGARSS 2005, Seoul, 25-29 July 2005.

[4] Z.-S. Zhou, W. M. Boerner, M. Sato, "Development of a broadband ground based polarimetric SAR system for noninvasive ground-truth validation in vegetation monitoring," IEEE Trans. Geosci. Remote Sensing, vol. 42, pp. 1803-1810, September 2004.

[5] L Sagues, J M Lopez, J Fortuny, X Fabregas, A Broquetas, A J Sieber, "Indoor experiments on polarimetric SAR interferometry," IEEE Trans. Geosci. Remote Sensing, vol. 38, pp. 670-684, March 2000.

[6] J.-R. J. Gau, and W. D. Burnside, "New polarimetric calibration technique using a single calibration dihedral," IEE Proc. Microw. Antennas Propag., vol. 142, no. 1, pp. 19-25, February 1995.

[7] S. R. Cloude, J. Fortuny, J. M. Lopez-sanchez, "Wide-band polarimetric radar inversion studies for vegetation layers," IEEE Trans. Geosci. Remote Sensing, vol. 37, pp. 2430-2441, September 1999.

[8] R. N. Treuhaft, S. R. Cloude, "The structure of oriented vegetation from polarimetric interferometry," IEEE Trans. Geosci. Remote Sensing, vol. 37, pp. 2620-2624, September 1999.

[9] A. Monakov, J. Vivekanandan, A. Stijernman and A. Nystrom, "Spatial and frequency averaging techniques for a polarimetric scatterometer system," IEEE Trans. Geosci. Remote Sensing, vol. 32, pp. 187-196, January 1994.

[10] R. N. Treuhaft, P. Siqueria, "Vertical structure of vegetated land surfaces from interferometric and polarimetric radar," Radio Science, vol. 35, pp. 141-177, Jan. 2000.

[11] S. R. Cloude, "Polarisation coherence tomography," Radio Science, in press. 